\title{
Ocorrência de Rickiella edulis (Speg.) Pfister (Pezizomycetes, Ascomycota) no Sudeste brasileiro
}

\author{
Larissa Trierveiler-Pereira ${ }^{1,3}$, Filipe Rochel de Almeida ${ }^{2}$ e (D) Juliano Marcon Baltazar ${ }^{1}$
}

Recebido: 14.04.2020; aceito: 07.07.2020

Como citar: Trierveiler-Pereira, L., Almeida, F.R. \& Baltazar, J.M. 2020. Ocorrência de Rickiella edulis (Speg.) Pfister (Pezizomycetes, Ascomycota) no Sudeste brasileiro. Hoehnea 47: e342020. http://dx.doi.org/10.1590/2236-8906-34/2020.

\begin{abstract}
Ocurrence of Rickiella edulis (Speg.) Pfister (Pezizomycetes, Ascomycota) in Southeastern Brazil). Rickiella edulis (Speg.) Pfister is an unique species of Pezizales due to the lacunose external surface of the apothecia. It is found on rotten wood and its occurrence is reported from Paraguay, Southern Brazil and Argentina. Specimens of $R$. edulis from the State of São Paulo are the first collections of the species in Southeastern Brazil. The aim of this study is to present a description and field photographs of the species and to update its distribution in South America.
\end{abstract}

Keywords: apothecia, macrofungi, neotropical fungi, Sarcoscyphaceae

RESUMO - (Ocorrência de Rickiella edulis (Speg.) Pfister (Pezizomycetes, Ascomycota) no Sudeste brasileiro). Rickiella edulis (Speg.) Pfister é uma espécie única de Pezizales devido à superfície externa lacunosa dos apotécios. A espécie é encontrada sobre madeira em decomposição e sua ocorrência é registrada para o Paraguai, sul do Brasil e Argentina. Espécimes de $R$. edulis coletados no Estado de São Paulo correspondem ao primeiro registro da espécie na região Sudeste do país. O objetivo desse trabalho é apresentar descrição e fotografias de campo dos espécimes coletados, assim como atualizar o conhecimento sobre a distribuição da espécie na América do Sul.

Palavras-chave: apotécio, fungos neotropicais, macrofungos, Sarcoscyphaceae

\section{Introdução}

A família Sarcoscyphaceae Le Gal ex Eckblad (Pezizales, Ascomycota) inclui espécies saprófitas que produzem ascomas epígeos, ou seja, se desenvolvem sobre madeira em decomposição ou outras partes de plantas mortas. Os ascomas geralmente são cupulados (apoteciais) e de cores vivas, podendo ser sésseis ou estipitados (Pfister 2015, Pfister et al. 2020). De acordo com Ortega-López et al. (2019), a família é bastante numerosa e inclui 13 gêneros e mais de 100 espécies. Essa família comporta gêneros de ascomicetos com espécies comumente encontradas em matas brasileiras, como espécies de Phillipsia Berk. e Cookeina Kuntze (observação de campo dos autores).

No Brasil, espécimes lignícolas com ascomas cupuliformes bastante peculiares foram coletados por J. Rick no Estado do Rio Grande do Sul no início do século
XX. Esse material foi enviado a Sydow, que propôs um novo gênero: Rickiella Syd. \& P. Syd. A nova espécie foi batizada Rickiella transiens Syd. \& P. Syd. (Rick 1904). Posteriormente, Pfister (1978) analisou o material tipo e concluiu que se tratava da mesma espécie que Peziza edulis Speg., descrita anteriormente para o Paraguai (Spegazzini 1891). Além da sinonímia, Pfister propôs a combinação de P. edulis para Rickiella.

Após análises moleculares provenientes de espécimes do norte da Argentina, Romero et al. (2012) concluíram que Rickiella pertence à família Sarcocyphaceae e se trata de um gênero próximo, porém distinto, de Phillipsia, posicionamento anteriormente defendido por Korf (1983).

Recentemente, $R$. edulis foi incluída na categoria "Em perigo" (EN) segundo os critérios de avaliação da União Internacional para a Conservação da Natureza e dos Recursos Naturais (IUCN) (Calle et al. 2020). No Brasil, a espécie

1. Universidade Federal de São Carlos, Centro de Ciências da Natureza, Campus Lagoa do Sino, Rodovia Lauri Simões, km 12, SP-189, 18290-000 Buri, SP, Brasil

2. Faculdade de Tecnologia de Itapetininga, Rua João Vieira Camargo, 104, Vila Barth, 18205-600 Itapetininga, SP, Brasil

3. Autor para correspondência: Lt_pereira@yahoo.com.br 
era, até o presente, conhecida apenas para o Estado do Rio Grande do Sul.

Pela primeira vez, R. edulis é coletada na região Sudeste do país, no Estado de São Paulo. Sendo assim, o intuito desse estudo é ampliar o conhecimento sobre a distribuição geográfica da espécie e apresentar descrição e fotografias do material coletado.

\section{Material e métodos}

Os espécimes foram coletados em mata ciliar do Rio Guareí no município de Angatuba (2329’24”S, 48²4’46”O), sudoeste do Estado de São Paulo, Brasil. Angatuba encontrase em um local interessante do ponto de vista ecológico, pois está situada em uma região de transição entre os biomas da Mata Atlântica (Floresta Estacional Semidecidual) e do Cerrado. A hidrografia do município é composta por três rios: rio Guareí, rio Itapetininga e rio Capivari, pertencentes à bacia hidrográfica do Alto Paranapanema.

Os ascomas foram fotografados in situ e retirados do substrato com auxílio de canivete para posterior análise laboratorial. Os ascomas foram examinados macro e micromorfologicamente, e posteriormente desidratados em estufa elétrica de circulação de ar. Os códigos apresentados para as cores das estruturas seguiu o trabalho de Kornerup \& Wanscher (1978). Para a análise microscópica, pequenas porções do himênio foram seccionadas a mão livre com lâmina de aço e a lâmina de vidro para microscopia foi montada com hidróxido de potássio $(\mathrm{KOH})$ e corante floxina $1 \%$.

O material estudado encontra-se depositado em dois herbários brasileiros: na Coleção Micológica do Herbário SPSC (Universidade Federal de São Carlos, Buri, SP), com duplicata no Herbário SP (Instituto de Botânica, São Paulo, SP).

\section{Resultados e Discussão}

Rickiella edulis (Speg.) Pfister, Mycotaxon 29: 330 (1987). $\equiv$ Peziza edulis Speg., Revista Argent. Hist. Nat. 1(6): 422 (1891).

$=$ Rickiella transiens Syd. \& P. Syd. ex Rick, Annls mycol. 2(3): 244 (1904).

Figura 1
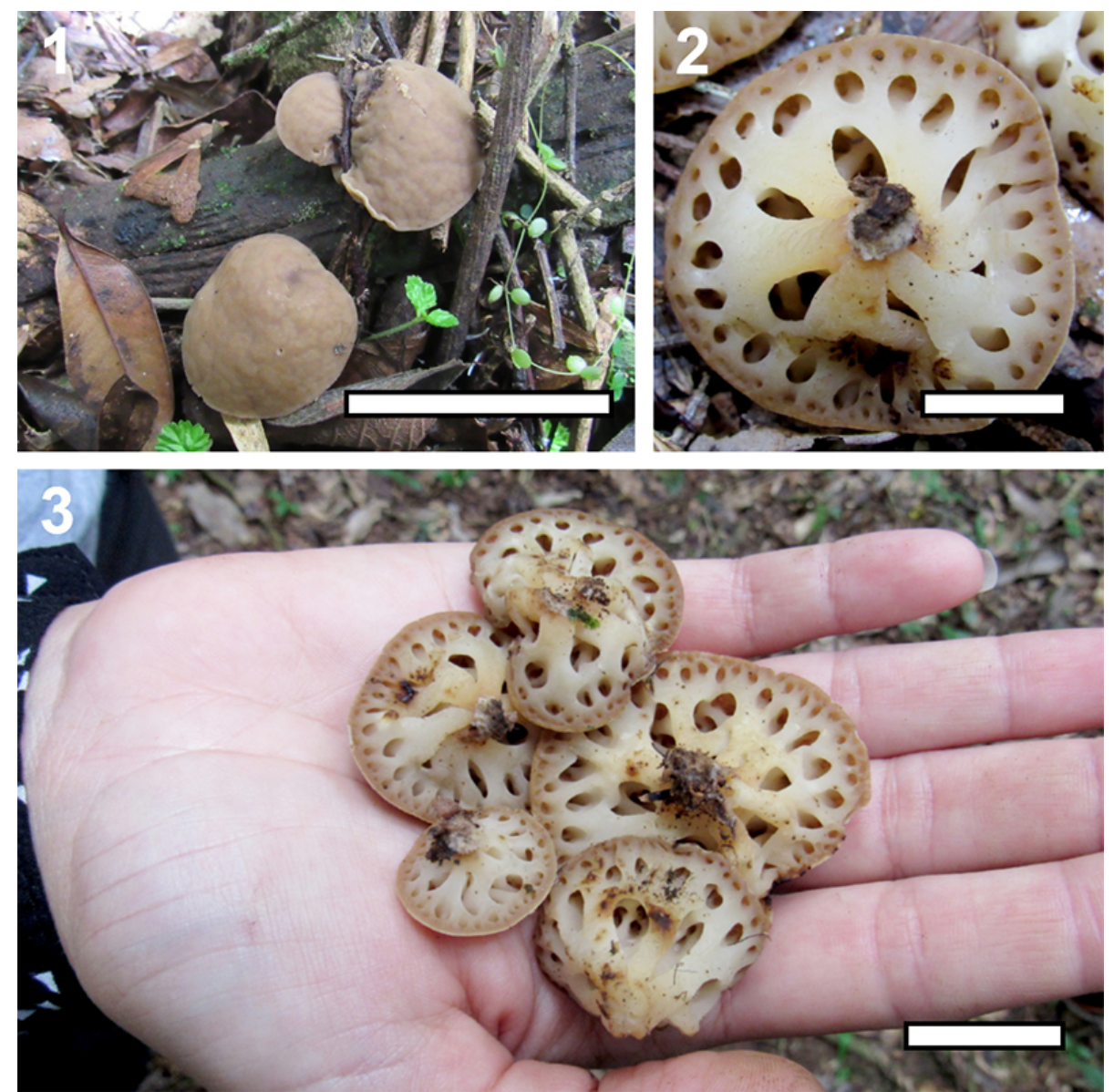

Figura 1. Rickiella edulis (Speg.) Pfister. 1. Ascomas in situ, sobre madeira em decomposição (escala = 5,0 cm). 2 . Visão inferior do ascoma $($ escala $=1,0 \mathrm{~cm}) .3$. Ascomas ex situ $($ escala $=2,0 \mathrm{~cm})$.

Figure 1. Rickiella edulis (Speg.) Pfister. 1. Ascomes in situ, on rotten wood (scale $=5.0 \mathrm{~cm})$. 2. Inferior view of ascome $(\mathrm{scale}=1.0$ $\mathrm{cm})$. 3. Ascomes ex situ $($ scale $=2.0 \mathrm{~cm})$. 
Ascomas 2-4,5 cm de diâm. $\times$ 1-2,5 cm alt., convexo, substipitado. Superfície himenial lisa, marrom alaranjada (6C5) a marrom clara (6D5), com linhas reticuladas que correspondem às sombras dos canais da superfície inferior, consistência subcartilaginosa, espessura da camada fértil $0,5 \mathrm{~mm}$. Superfície inferior amarelo pálida (1AC) a cinza amarelado (4B2), lacunar, com canais.

Ascosporos elipsoides, hialinos, lisos, 19-23 × 10-14 $\mu \mathrm{m}$, de paredes levemente espessadas, geralmente bigutulados. Ascos cilíndricos, hialinos, contendo 8 ascóporos, de 11-14 $\mu \mathrm{m}$ de diâm., de paredes levemente espessadas. Paráfises retas, hialinas, septadas, 2-4 $\mu \mathrm{m}$ de diâm., com ápice cilíndrico, não-dilatado. Hifas do subhimênio aglutinadas, hialinas a amareladas, septadas, 2-6 $\mu \mathrm{m}$ de diâm.

Material examinado: BRASIL. S̃̃o PAULO: Angatuba, Bairro Guareí Velho, mata ciliar do Rio Guareí, 29-II-2020, F.R. Almeira \& L. Trierveiler-Pereira s.n. (SPSC, SP dupl.).

Habitat: ascomas gregários sobre madeira em decomposição.

Distribuição conhecida: Norte da Argentina (La Caldera, Província de Salta), Região Central do Paraguai (Guarapí, Departamento de Paraguarí), Sul e Sudeste do Brasil (Estados do Rio Grande do Sul e São Paulo).

Comentários: As principais características para identificação da espécie são a coloração amarronzada da superfície himenial, a superfície inferior cavernosa de coloração amarelada, e o hábito lignícola. Os ascósporos são elipsoides e hialinos, de paredes lisas e levemente espessadas, conforme ilustrado por Pfister (1978).

Os espécimes examinados foram encontrados no interior de mata, em local sombreado. Spegazzini (1891) descreve que o material original foi coletado sobre solo, mas provavelmente estava sobre madeira em estágio muito avançado de decomposição, já que todos os outros registros da espécie são sobre madeira.

A espécie recebeu o epíteto "edulis" porque, segundo a descrição original, é comestível. Porém, não há outros registros ou mais detalhes sobre esse fato na literatura.

No Brasil, a espécie havia sido registrada apenas no Rio Grande do Sul. Alguns materiais brasileiros estão depositados em herbários nacionais e internacionais como Rickiella transiens, sinônimo de Rickiella edulis segundo Pfister (1978). Este é o primeiro registro da espécie na região Sudeste do Brasil, ampliando assim a distribuição geográfica de $R$. edulis na região sul-americana.

\section{Agradecimentos}

Os autores agradecem ao Conselho Nacional de Desenvolvimento Científico e Tecnológico (CNPq), pelo auxílio financeiro (Chamada Universal 01/2016, $\mathrm{n}^{\circ}$ processo 422514/2016-0); e aos membros da ONG EcoRoad (Angatuba, São Paulo), pelo auxílio nas coletas.

\section{Literatura citada}

Calle, A., Drechsler-Santos, E.R., Funez, L., Kossmann, T., da Cunha, K.M., Pfister, D., Robledo, G., SandovalLeiva, P., Sanjuan, T. \& Vasco-Palacios, A. 2020. Rickiella edulis. The IUCN Red List of Threatened Species 2020: e.T172818281A172861317.

Korf, R.P. 1983. Cyttaria (Cyttariales): Coevolution with Nothofagus and evolutionary relationship to the Boedijnopezizeae (Pezizales, Sarcoscyphaceae). Australian Journal of Botany: Supplementary Series 10: 77-87.

Kornerup, A. \& Wanscher, J.H. 1978. Methuen handbook of colour. Londres: Eyre Methuen.

Ortega-López, I., Valenzuela, R., Gay-González, A.D., Lara-Chávez, M.B.N., López-Villegas, E.O. \& Raymundo, T. 2019. La familia Sarcoscyphaceae (Pezizales, Ascomycota) en México. Acta Botanica Mexicana 126: e1430.

Pfister, D.H. 1978. The placement of Peziza edulis in Rickiella (Sarcoscyphaceae, Pezizales). Mycotaxon 29: 329-333.

Pfister, D.H. 2015. Chapter 2. Pezizomycotina: Pezizomycetes, Orbiliomycetes. In Mclaughlin, D.J., Spatafora, J.W. (eds): The Mycota VII (B). Systematics and Evolution. Berlim: Springer-Verlag, pp. 35-55.

Pfister, D.H., Quijada, L. \& LoBuglio, K.F. 2020. Geodina (Pezizomycetes: Wynneaceae) has a single widespread species in tropical America. Fungal Systematics and Evolution 5: 131-138 (no prelo).

Rick, J. 1904. Über einige neue und kritische Pilze SüdAmerikas. Annales Mycologici 2(3): 242-247.

Romero, A.I., Robledo, G., LoBuglio, K.F. \& Pfister, D.H. 2012. Rickiella edulis and its phylogenetic relationships within Sarcoscyphaceae. Kurtziana 37(1): 79-89.

Spegazzini, C. 1891. Fungi guaranitici nonnulli novi vel critici. Revista Argentina de Historia Natural 1(6): 398432. 